Research Article

Open Access

\title{
The Polymorphism of Osteopontin Gene and Receptors are associated with Allergic Rhinitis in a Chinese Han Population
}

\author{
Zhihai Zhang, Xia Ke, Yang Shen, Houyong Kang ${ }^{\star}$ and Suling Hong* \\ Professor, Department of Otorhinolaryngology, First Affiliated Hospital of Chongqing Medical University, Chongqing, China
}

\section{Article Info}

*Corresponding authors:
Suling Hong
Professor
Department of Otorhinolaryngology
The First Affiliated Hospital of Chongqing
Medical University
Chongqing, China
E-mail: 489272017@qq.com

\section{Houyong Kang}

Department of Otorhinolaryngology

The First Affiliated Hospital of Chongqing

Medical University

Chongqing, China

E-mail: drkexia@163.com

Received: April 25, 2017

Accepted: May 29, 2017

Published: June 5, 2017

Citation: Zhang Z, Ke X, Shen Y, Kang H, Hong S. The Polymorphism of Osteopontin Gene and Receptors are associated with Allergic Rhinitis in a Chinese Han Population. Madridge J Immunol. 2017; 1(1): 5-9. doi: $10.18689 / \mathrm{mjim}-1000102$

\section{Copyright: (c) 2017 The Author(s). This work is licensed under a Creative Commons Attribution 4.0 International License, which permits unrestricted use, distribution, and reproduction in any medium, provided the original work is properly cited.}

Published by Madridge Publishers

\begin{abstract}
Osteopontin (OPN) is a pro inflammatory cytokine involved in chronic inflammatory diseases. In this study, we evaluated the potential association of OPN polymorphisms with Allergic Rhinitis (AR) in a Chinese Han population. Three single-nucleotide polymorphisms (SNPs) in OPN-rs9138, rs1126616, rs1126772, and eight SNPs of its receptor-rs1448623, rs3770136, rs3738919, rs3911238, rs5918, rs2056131, rs187116, rs 16927061 were genotyped in 1020 AR patients and 1173 healthy controls by using a PCR-restriction fragment length polymorphismassay. The allele, genotype, and haplotype frequenciesin the patients and the controls were compared using a $\chi 2$ test. Moreover, we performed haplotype analysis by using the online software platform SHE sis. The results revealed a significant association between five SNPs-rs9138, rs1126616, rs1126772, rs1449263, rs187116-and AR in the studied Chinese Han population. Furthermore, the ATG, CCG haplotype were associated with a significantly higher prevalence of AR than were the CTA haplotypes. However, no significant differences were detected in either the genotype or allele frequencies of the other SNPs between the AR and control groups. Overall, this study has identified a strong association between OPN polymorphisms and increased risk of AR in a Chinese Han population.
\end{abstract}

Keywords: OPN; Allergic rhinitis; SNP.

Allergic rhinitis (AR) is an inflammatory disease of the nasal mucosa mediated by $\lg \mathrm{E}$, characterized by 4 major symptoms: nasal congestion, rhinorrhoea, nasal itching, and sneezing. It affects $10-20 \%$ of the population worldwide [1]. For the prevalence of ARin China, it has increased markedly over the last 2 decades, especially in western regions [2]. For example, the overall incidence of AR in Chongqing in 2008 was estimated to be $32.3 \%$, and is predicted to increase in the following years.

Currently, environment and gene are regarded as the main etiologic factors of AR. For environmental factors, pollens, house dust mite maybe the most popular allergens. While for genetic predisposition, single nucleotide polymorphisms (SNPs) in numerous genes may play an important role $[3,4]$. Genes such as IL-23R, MRPL4, and TNF- $\alpha$ have been reported relevant to the susceptibility to AR $[5,6]$.

Osteopontin (OPN) is a Th1 cytokine with multiplefunctions in inflammation, immunity, and bone metabolism. Recent studies have reported that persistence of OPN expression may exacerbate autoimmune or inflammatory diseases such as multiple sclerosis (MS) [7]. Crohn's disease [8]. systemic lupus erythematosus (SLE) [9] and rheumatoid arthritis (RA) [10]. In addition, genetic polymorphisms of OPN and its receptors were also reported to be associated with RA [11], MS [12,13], asthma [14,15, 16], and SLE [17]. While whether theyare involved in the development of AR is not yet known and were therefore the subject of the current study. 


\section{Materials and Methods}

\section{Ethics statement}

This study was approved by the Ethics Committee of the First Affiliated Hospital of Chongqing Medical University. Written informed consent was obtained from all adult participants and from parents or legal guardians of minors.

\section{Patients}

From May 2011 to June 2014, 1020 AR patients aged 18 to 60 years were enrolled in this study. All patients were identified by and treated at the outpatient clinic of the Department of Otolaryngology at the First Affiliated Hospital of Chongqing Medical University, Chongqing, China. The diagnosis of AR was based on the patients' medical history, symptoms and the presence of a positive skin pricking test (SPT, Allergopharma, Hamburg, Germany) in response to a panel of common allergens defined by the ARIA 2008 guidelines [18]. The SPT results were diagnosed in accordance with the recommendations of the Subcommittee on Allergen Standardization and Skin Tests of the European Academy of Allergy and Clinical Immunology [19].

A positive SPT result was defined as the formation of a wheal larger than or equal to one half the diameter of the histamine control wheal, and at least $3 \mathrm{~mm}$ larger than the diameter of the negative control wheal. A total of 18 inhaled allergens were tested, including house dust, grass, tree, mold, food, and cat and dog dander et al. In contrast, patients with accompanying systemic disease were excluded from the study. A total of 1173 hereditarily-unrelated healthy individuals were enrolled as controls. They did not show clinical features or family history of allergies and had not experienced an upper respiratory tract infection within the 4 weeks prior to the study. All subjects of this study were of Chinese Han ethnic origin and from the Chongqing city of China.

\section{SNP selection and geno typing}

We studied 3 SNPs (rs9138, rs1126616, rs1126772, ) in human OPN gene, which have been documented to be associated with several immune diseases. Mean while, we also chose its receptors'SNP sintegrina4 (ITGA4) - rs1149263 and rs3770136, integrin $\alpha \mathrm{v}(\mathrm{ITGAV})$ - rs3738919 and rs3911238, integrin $\beta 3$ (ITGB3) -rs5918 and rs2056131, and CD44 -rs187116 and rs16927061 for gene polymorphism analysis.

The 11 SNPs were genotyped by restriction fragment length polymorphism (RFLP) analysis. Briefly, peripheral blood samples were collected from each subject and stored at $-80^{\circ} \mathrm{C}$ for further use. Genomic DNA was isolated from blood leukocytes using the Qiagen DNA Blood Mini kit (Qiagen, Valencia, CA, USA). Amplification of target DNA was performed by PCR using the primers listed in Table 2. PCR conditions were as follows: initial denaturation at $95^{\circ} \mathrm{C}$ for $5 \mathrm{~min}, 37$ cycles of denaturation at $95^{\circ} \mathrm{C}$ for $30 \mathrm{~s}$, annealing at $58-62^{\circ} \mathrm{C}$ for $30 \mathrm{~s}$, and extension at $72^{\circ} \mathrm{C}$ for $30 \mathrm{~s}$, and final extension at $72^{\circ} \mathrm{C}$ for $5 \mathrm{~min}$. The PCR products were digested with specific restriction enzymes (Table 2 )at $37^{\circ} \mathrm{C}$ for at least $4 \mathrm{~h}$. Digestion products were visualized on a $4 \%$ a garose gel and stained with Goodview (SBS Gene tech, Beijing, China). Genotypes were confirmed by direct sequencing by the Invitrogen Biotechnology Company (Guangzhou, China) using $20 \%$ of randomly selected PCR products.

\section{Statistical analysis}

Call rates for the SNPs studied were compared between AR patients and controls by the $\chi 2$ test. The $\chi 2$ test was also applied to compare demographic characteristics and the allele and genotype frequencies between the patients and controls. Estimation of genotype frequencies was performed by direct counting. The online software platform SHE sis was used to analyze the haplotype and probabilities. We used non-risk alleles as the reference, and tested all the other haplotypes. Logistic regression analysis was used to analyze the genotype allele, controlling for age, gender, and occupation as the co variables. Odds ratios (OR) and 95\% confidence intervals $(\mathrm{Cl})$ were calculated to determine the association of SNPs with the risk of AR. Statistical analysis was performed using the Statistical Package for the Social Sciences (SPSS) version 13.0 for Windows (SPSS Inc., Chicago, III. USA). $P$ values were corrected $(P C)$ for the number of alleles tested using the Bonferroni correction method. A Pc value of $<0.05$ was considered statistically significant.

\section{Results}

\section{Subject characteristics}

Demographic and clinical characteristics of the participants are presented in Table 1. The patient group consisted of 493 males and 527 females, with a mean age of 31.58 years (SD = 7). The control subjects included 593 males and 580 females and had a mean age of 33.23 years $(S D=8)$. There was no significant difference between the 2 groups with respect to mean age and gender distribution. About $51.8 \%$ of the patients were allergic to house dust mite, $18.1 \%$ to pollens, and $30.1 \%$ to mixed allergens.

\section{Genetic polymorphisms of OPN and its receptors in AR}

The genotype distributions of the 11examined SNPs in the OPN gene and its receptors were in Hardy-Weinberg equilibrium in both the $A R$ and control groups. The call rate for each SNP was $100 \%$. There were no statistically significant differences in the proportions of missing genotype data between cases and controls $(P>0.05)$. The results of genotypic and allelic frequency analysis are shown in Table 3 and 4.

For OPN, A significantly increased frequency of the $A C$, AA genotype and A allele of rs9138 in AR patients $\left(p=6.761 \times 10^{-3}\right.$. $\mathrm{OR}=1.281, \quad 95 \% \mathrm{Cl} \quad 1.072-1.531 ; \quad \mathrm{p}=6.693 \times 10^{-7} . \mathrm{OR}=2.117$, $95 \% \mathrm{Cl} 1.570-2.853 ; \mathrm{p}=4.39 \times 10^{-7} . \mathrm{OR}=1.257,95 \% \mathrm{Cl} 1.150-1.374$ ) (Table 3) were identified.

The results also showed obvious differences between the $A R$ patients and control patients concerning the frequencies of rs1126616. A higher frequency of the CC, CT genotype $\left(p=1.391 \times 10^{-6} \mathrm{OR}=2.111,95 \% \mathrm{Cl} 1.566-2.846 ; p=1.705 \times 10^{-3}\right.$ 
$\mathrm{OR}=1.337,95 \% \mathrm{Cl} 1.118-1.598)$ and the $C$ allele $\left(\mathrm{p}=2.834 \times 10^{-7}\right.$. $\mathrm{OR}=1.260,95 \% \mathrm{Cl} 1.376-1.53)$ were found in $\mathrm{AR}$ patients compared with the controls.

Meanwhile, a marked difference of AG, GG genotype and $G$ allele of rs1126772 were identified and a higher frequency can be observed in AR patients $\left(p=5.111 \times 10^{-5} . O R=1.442\right.$, $95 \% \mathrm{Cl} 1.209-1.179 ; p=1.106 \times 10^{-3} . \mathrm{OR}=1.793,95 \% \mathrm{Cl} 1.285-$ 2.555; $\mathrm{p}=4.231 \times 10^{-6} . \mathrm{OR}=1.260,95 \% \mathrm{Cl} 1.142-1.390$ ) (Table 3).

For the receptor ITGA4, there were significant differences concerning the frequencies of SNPs rs1449263. Comparing with the controls, $A R$ patients showed a significantly higher prevalence of the $A A, A G$ genotype $\left(p=1.597 \times 10^{-4} . O R=1.637\right.$, $\left.95 \% \mathrm{Cl} 1.263-2.121 ; p=1.525 \times 10^{-3} . \mathrm{OR}=1.341,95 \% \mathrm{Cl} 1.113-1.615\right)$ and $A$ allele $\left(p=4.868 \times 10^{-5} . O R=1.163,95 \% C l\right.$ 1.081-1.251).

When it comes to the receptor CD44, AR patients had a significantly increase in the frequencies of CT,TT genotype $\left(p=8.217 \times 10-3.0 R=1.289,95 \% C l\right.$ 1.070-1.552; $p=3.618 \times 10^{-4}$. $\mathrm{OR}=1.598,95 \% \mathrm{Cl} 1.236-2.066)$ and $\mathrm{T}$ allele $\left(\mathrm{p}=1.831 \times 10^{-4}\right.$. $\mathrm{OR}=1.149,95 \% \mathrm{Cl}$ 1.068-1.235) in rs187116. However, he other 6 SNPs of OPN receptors did not display significant associations with AR (Table 4).

\section{Haplotype analysis of OPN allelic variants}

A haplo type analysis was performed using the Haplo view V3.32 program and the SHE sis online software platform. The eight possible haplotype frequencies are shown in Table 5. The ATG and CCG haplotypes accounted for $7.0 \%$ and $7.6 \%$ of the AR patients respectively, and these were significantly higher in the patients than in controls $\left(P=6.69 \times 10^{-5}, \mathrm{OR}=\right.$ $1.700,95 \% \mathrm{Cl}=1.307-2.213 ; \mathrm{P}=2.16 \times 10^{-4}, \mathrm{OR}=1.594,95 \%$ $\mathrm{Cl}=1.243-2.044$ respectively). We also found that the CTA haplotypes were significantly less prevalent in AR patients ( $P$ $=5.00 \times 10^{-15}, \mathrm{OR}=0.593,95 \% \mathrm{Cl}=0.523-0.673$ ).

\section{Discussion}

OPN, which was originally identified as a bone matrix protein, was subsequently identified as a cytokine (Eta-1) produced by activated T cells and transformed cells [20,21,22]. As a matri cellular protein, Osteopontin (OPN) is expressed in many different cell types, including dendritic cells, T cells, B cells and so on [23]. As a cytokine, OPN can stimulate T cell proliferation, interfere with the differentiation of $\mathrm{T}$ cells into Th1 or Th2 and regulate immunoreactions [24,25,26]. Receptors of OPN include certain integrins $[27,28,29]$ and CD44 variants [30,31,32]. With the potential to interact with multiple ubiquitously expressed cell surface receptors, OPN plays active roles in many physiological and pathological processes including wound healing, inflammation, immune response and so on.

So far, OPN has been identified as a biomarker for various types of inflammatory diseases and cancers [33,34] dys regulated and excessive expression of OPN have also been linked to some of the autoimmune disorders such as SLE [35], MS [36], RA [37]. chronic obstructive pulmonary disease [38], and asthma [39]. It has been reported that OPN can stimulate the production of the Th1 cytokine interferon- $\gamma$ and the Th17 cytokine IL-17 and inhibited the Th2 cytokine IL-10 [23,40]. This was further identified by a recently study, which demonstrated that an increased Th1/Th2 ratio and Th17 cells have been involved in the pathogenesis of Behcet's disease by OPN. So in this study we try to make sure whether OPN play a role in the pathogenesis $A R$, which is the most popular allergic disease in the upper airway.

We focused on the polymorphisms of the OPN gene and the susceptibility to AR. The selection of the SNPs of OPN was based on earlier reports $[13,17,41]$. Meanwhile, because OPN exerts its effects through interacting with multiple receptors, the polymorphisms of these receptors were also investigated $[11,12]$.

Our data demonstrated the association of SNPs of rs9138, rs1126616, rs1449623, rs1448623, rs3770136, rs3738919, rs3911238, rs5918, rs2056131, rs187116, rs16927061 in the OPN gene and its receptors ITGA4, ITGAV, ITGB3, CD44 with $A R$. In the case of $r 59138$, the $A A, A C$ genotypeand the $A$ allele significantly increased the risk of AR. Meanwhile, the $C T, C C$ genotype and $C$ allele of rs 1126616 was also identified as an $A R$ risk factor. For rs 1126772 , the $A G, G G$ genotype and $G$ allele also significantly increased the risk of $A R$. When it comes to the receptors of OPN, only the polymorphisms of ITGA4 and CD44 showed relevance. For rs1449623 of ITGAV4, the $A R$ group had an increase in AG, AA genotype and $A$ allele. We alsodetected a risk association between rs187116 of CD44 and $A R$ patients with the $C T$, TT genotype and T allele.The study has revealed two clinical clues related to AR. First, ourresults identified that three OPN SNPs (rs9138, rs1126616, andrs1126772) and two OPN receptor SNPs (rs1449263 of ITGA4 and rs187116 of CD44) may strongly associated with the risk of $A R$ in Chinese Han patients. Furthermore, the haplo type analysis performed here revealed that the OPN haplotypes ATG and CCGwas significantlymoreprevalent in patientswith AR than in controls. While haplo types of CTA was negatively relevant to AR. Thus we suggest that these SNPs may be chosen as tag SNPs to evaluate thehigh risk of developing AR in the Chinese Han population.

In the present study, to decrease the influenceof confounding factors on the results, we selected the AR patients and controls using strict guidelines and confirmed the genotyping results by direct sequencing. And we observed a novel association between SNPs of OPN and AR in the Chinese Han population. However, it is worth mentioning that there are some limitations to our study. First, we did notdetect the protein level of OPN and receptors in the peripheral blood and perform a functional analysis study, so we could not draw a certain conclusion about the influence of these polymorphisms on the cytokine levels. Second, as it is well known that environmental factors are critical for the development of $A R$, further studies will be needed to clarify the genetic influence of OPN in AR pathogenesis, including gene-gene and gene-environment interactions. 
In conclusion, this study provides further evidence of the association of OPN and receptors with $A R$, suggesting the possible involvement of these genes in the susceptibility to AR. This suggests that combining information from common risk polymorphisms could improve disease prediction. Further functional studies of genetic variants could contribute to an increased understanding of the roles of the studied genes in the pathogenesis of $A R$.

\section{Acknowledgments}

This study was supported by the National Natural Science Foundation of China (81300811) and National Key Clinical Specialties Construction Program of China.

\section{Author Contributions}

Z.Z. and X.K. devised the concept, designed the experiment, and wrote the manuscript. Y.S. and H.K. performed the experiments and analyzed the data. S.H. supervised the project. All authors discussed the results and contributed in this manuscript.

\section{Disclosures}

No conflicts of interest, financial or otherwise, are declared by the author(s).

\section{References}

1. Togias A. Rhinitis and asthma: evidence for respiratory system integration. J Allergy Clin Immunol. 2003; 111(6): 1171-83. doi: 10.1067/ mai.2003.1592

2. Shen J. Epidemiological features of allergic rhinitis in four major cities in Western China. J Huazhong Univ Sci Technolog. Med Sci. 2011; 31(4) : 433-40. doi: 10.1007/s11596-011-0469-1

3. Gaddam SL. Association of interleukin-10 gene promoter polymorphism in allergic patients. Genet Test Mol Biomarkers. 2012; 16: 632-635. doi: 10.1089/gtmb.2011.0255

4. Ibrahim GH. Interleukin-18 gene polymorphisms in Egyptian patients with allergic diseases. Am J Rhinol Allergy. 2012; 26 (5): 385-389. doi: 10.2500/ajra.2012.26.3806

5. Hu D. Association betweenpolymorphisms of the IL-23R gene and allergic rhinitis in a Chinese Han population. PLoS One. 2013; 8(5): e63858.

6. Wei $X$. The association between polymorphisms in the MRPL4 and TNF- $\alpha$ genes and susceptibility to allergic rhinitis. PLoS One. 2013; 8(3): e57981.

7. Comabella M. Plasma osteopontinlevels in multiple sclerosis. J Neuro immunol. 2005; 158: 231-9. doi: 10.1016/j.jneuroim.2004.09.004

8. Agnholt J. Osteopontin, a protein with cytokine-like properties, is associated with inflammation in Crohn's disease. Scand J Immuno. 2007: I65: 53-60. doi: 10.1111/j.1365-3083.2007.01908.x

9. Wong CK. Elevation of plasma osteopontin concentration is correlated with disease activity in patients with systemic lupus erythematosus. Rheumatology. 2005; 44: 602-6. doi: 10.1093/keh558

10. Sennels $H$. Circulating levels of osteopontin, osteoprotegerin, total soluble receptor activator of nuclear factor-kappa B ligand, and highsensitivity $C$-reactive protein in patients with active rheumatoid arthritis randomized to etanercept alone orin combination with methotrexate. Scand J Rheumatol. 2008; 37: 241-7.
11. Hollis, Moffatt JE. The ITGAV rs 3738919 variant and susceptibility to rheumatoid arthritis in four Caucasian sample sets. Arthritis Res Ther. 2009; 11: 152 .

12. O'Dohert C. ITGA4 polymorphisms and susceptibility to multiple sclerosis. J Neuro immunol. 2007; 189: 151-7. doi: 10.1016/j.jneuroim.2007.07.006

13. Niino M. Genetic polymorphisms of osteopontin in association with multiple sclerosis in Japanese patients. J Neuro immunol. 2003; 136 125-9.

14. Weiss LA. Variation in ITGB3 is associated with asthma and sensitization to mold allergen in four populations. AmJ Respir Crit Care. 2005; 172: 67-73.

15. Thompson EE. Integrin beta 3 genotype influences asthma and allergy phenotypes in the first 6 years of life. J Allergy Clin Immunol. 2007; 119: 1423-29.

16. Mathias RA. A graphical assessment of $p$-values from sliding window haplotype tests of association to identify asthma susceptibility loci on chromosome 11q. BMC Genet. 2006; 7: 38. doi: 10.1186/1471-2156-7-38

17. D'Alfonso $\mathrm{S}$. Two single-nucleotide polymorphisms in the $5^{\prime}$ and $3^{\prime}$ ends of the osteopontin gene contribute to susceptibility to systemic lupus erythematosus. Arthritis Rheum. 2005; 52: 539-47.

18. Bousquet J. Allergic rhinitis and its impact on asthma (ARIA) 2008 update (in collaboration with the World Health Organization, GA(2)LEN and AllerGen). Allergy. 2008; 63: 8-160.

19. Allergen standardization and skin tests. The European Academy of Allergology and Clinical Immunology. Allergy. 1993; 48 (Suppl. 14) pp. 48-82.

20. Patarca R. Molecular and cellular basis of genetic resistance to bacterial infection: the role of the early $T$ lymphocyte activation-1/osteopontin gene. Crit Rev Immunol. 1993; 13: 225-46.

21. Sodek J. Osteopontin. Crit Rev Oral Biol. Med. 2000; 11: 279-303.

22. Denhardt DT. Osteopontin as a means to cope with environmental insults: regulation of inflammation, tissue remodeling, and cell survival. J Clin Invest. 2001; 107: 1055-61. doi: 10.1172/JCI12980

23. Wang KX, Denhardt DT, Osteopontin: role in immune regulation and stress responses. Cytokine Growth Factor Rev.2008; 19: 333-45.

24. Stromnes IM, Goveman, JM. Osteopontin-induced survival of T cells. $N$ at Immunol. 2007; 8 (1): 19-20.

25. Ashkar S, Weber GF. Eta-I (osteopontin) : an early component of typeI(cell-mediated)immunity. Science. 2000 ; 287 (5454): 860-4.

26. O'Regan AW. Osteopontin augmentsCD3-mediated interferon-gamma and CD40 ligandexpression by T cells, which results in II-12 production from peripheral blood mononuclear cells. J Leukoc Biol. 2000; 68 (4): 495-502.

27. O'Regan A, Berman JS. Osteopontin: a key cytokine in cell-mediated and granulomatous inflammation. Int J Exp Pathol. 2000; 81(6): 373-90. doi: 10.1046/j.1365-2613.2000.00163.x

28. Yokosaki $Y$. Distinct structural requirements for binding of the integrins alphavbeta6, alphavbeta3, alphavbeta5, alpha5 beta1 and alpha9 beta1 to osteopontin. Matrix Biol. 2005; 24: 418-27.

29. Tanani MK. The regulation and role of osteo pontin in malignant transformation and cancer. Cytokine Growth Factor Rev. 2006; 17: 46374. doi: 10.1016/j.cytogfr.2006.09.010

30. Weber GF. Receptor-ligandinteraction between CD44 and osteopontin (Eta-1). Science. 1996; 271: 509-12.

31. Katagiri YU. CD44 variants but not CD44 s cooperate with beta1containingintegrins to permit cells to bind to osteopontin independently of arginine-glycine-aspartic acid, thereby stimulating cell motility and chemotaxis. Cancer Res. 1999; 59: 219-26.

32. Sodek J. Osteopontin and mucosal protection. J Dent Res. 2006; 85: 40415.

33. Rangaswami $\mathrm{H}$, et al. Osteopontin: role in cell signaling and cancer 
progression. Trends Cell Biol. 2006; 16:79-87. doi: 10.1016/j.tcb.2005.12.005

34. Ramaiah SK, Rittling, S. Pathophysiological role of osteopontin in hepatic inflammation, toxicity and cancer. Toxi col Sci. 2008; 103: 4-13.

35. Zandman GG, Shoenfeld Y, SLE and infections. Clin Rev Allergy Immunol. 2003; 25: 29-40.

36. Steinman L, et al. Multiple sclerosis: deeper understanding of its pathogenesis reveals new targets for therapy. Annu Rev Neurosci. 2002; 25: 491-505.

37. Xu G, et al. Role of osteopontin in amplification and perpetuation of rheumatoid synovitis. J Clin Invest. 2005; 115(4): 1060-7. doi: 10.1172/ $\mathrm{JCl} 23273$

38. Woodruff PG, et al. A distinctive alveolar macro phage activation state induced by cigarette smoking. Respir Crit Care Med. 2005; 172: 383-92.

39. Xanthou, G.et al. Osteopontin has a crucial role in allergic airway disease through regulation of dendritic cell subsets. Nat Med. 2007; 13: 570-8. doi: $10.1038 / \mathrm{nm} 1580$

40. Murugaiyan $\mathrm{G}$, et al. Increased osteopontin expression in dendritic cells amplifies IL-17 production by CD4(+) T cells in experimental autoimmune encephalomyelitis and in multiple sclerosis. J Immunol. 2008; 181(11) 7480-8.

41. Forton AC, et al. Anosteopontin (SPP1) polymorphism is associated with systemic lupus erythematosus. Hum Mutat. 2002; 19: 459. doi: 10.1002/ humu.9025 